\title{
Tumor with slow-developing and recurring lumps of the external ear with skull base and lung metastasis: A case report
}

\author{
ZHIFANG ZHAI $^{1}$, JIE HU ${ }^{2}$, YI YOU ${ }^{1}$, XICHUAN YANG $^{1}$ and ZHIQIANG SONG ${ }^{1}$ \\ ${ }^{1}$ Department of Dermatology, The First Affiliated Hospital, Army Medical University, Chongqing 400038; \\ ${ }^{2}$ Department of Dermatology, The First Affiliated Hospital, Zunyi Medical College, Zunyi, Guizhou 563003, P.R. China
}

Received September 17, 2019; Accepted April 15, 2020

DOI: $10.3892 / \mathrm{ol} .2020 .11724$

\begin{abstract}
The present case report describes a rare case of recurring lumps on the external ear in a 46-year-old Chinese male with a history of $>10$ years, who presented with large irregular red lumps on the right temple and with lung and skull base metastasis. The patient had a lesion in the right auricle and experienced recurrence following surgical resection. A thorough systemic evaluation revealed no other obvious abnormalities. The patient was diagnosed with adenoid cystic carcinoma (ACC), a slow-growing form of high-grade adenocarcinoma, and refused any further treatment at the present hospital (The First Affiliated Hospital of Army Medical University). At 2 years post-follow-up, the patient had become more frail but was in good spirits. The present study indicated that ACC is a low-grade and commonly slow-developing malignancy that primarily arises from a salivary gland, with recurring and metastatic characteristics.
\end{abstract}

\section{Introduction}

Adenoid cystic carcinoma (ACC) is a rare type of cancer that occurs primarily in the major and minor salivary glands (up to 50 and $35 \%$, respectively), but it may also be located at other sites, including the oropharyngeal and nasopharyngeal spaces, external ear, trachea, breast, lacrimal gland, skin and lower female genital tract (1). ACC is most frequently detected in younger- and middle-aged adults, but individuals of any age may be affected, including children. Certain studies have determined a median age of $\sim 60$ years and a greater prevalence in female patients (up to $60 \%$ of cases) $(1,2)$. ACC usually presents in a localized manner and is limited to the primary

Correspondence to: Dr Xichuan Yang or Dr Zhiqiang Song, Department of Dermatology, The First Affiliated Hospital, Army Medical University, 30 Gaotanyan Street, Chongqing 400038, P.R. China

E-mail: drsongzq@hotmail.com

E-mail: doctoryxc@qq.com

Key words: adenoid cystic carcinomas, external ear, lung metastasis, skull base metastasis sites, but a small proportion of patients exhibit regional lymph node involvement and distant organ metastasis at diagnosis, particularly in the lungs. To date, the biological behavior of ACC has remained poorly understood, and clinically, it is primarily treated by surgery, with or without adjuvant radiation therapy.

\section{Case report}

A 46-year-old male patient visited the First Affiliated Hospital of Army Medical University (Chongqing, China) in May 2016 with recurring asymptomatic large irregular red lumps on the right temple for $\sim 6$ years. The patient also complained of slight breathlessness on exertion for half a year. The patient's medical history was provided by the patient and the patient's daughter. In early 2006, a slow-growing red nodule arose on the right auricle. It was gradually enlarged to the size of a pigeon egg and was surgically excised at a separate hospital (The First Affiliated Hospital of Chongqing Medical University; Chongqing, China) in January 2010. The histopathological diagnosis was ACC and there was no obvious evidence of spread elsewhere during the general physical examination. After a few months, indurated plaques appeared on the right temple and gradually enlarged over time. For economic reasons, the patient did not seek any medical help until April 2016. The patient refused operative treatment once again. In the recent half year, the tumors on the right temple increased markedly and the patient experienced slight breathlessness after activity and he then visited a hospital.

The patient was underweight (Body Mass Index, 16.7), but appeared reasonably energetic. Physical examination indicated no obvious abnormality besides the skin lesions. There was no associated cervical lymphadenopathy. On local examination, a solitary non-tender proliferative growth, measuring $\sim 5 \times 4 \mathrm{~cm}$ with an uneven surface and irregular margins, was arising on the right temple without erosion or bleeding. There were obviously dilated capillaries on the surface. The upper part of the right auricle was absent due to the previous surgery (Fig. 1).

An excision biopsy from the center of tumors on the right temple was performed. Histopathological examination revealed a neoplasm that was sharply demarcated from surrounding the normal parenchyma by reactive 
gliosis (Fig. 2A). The epithelial tumor cells proliferated with a tubular and cribriform pattern, in certain places forming ductular structures lined by stratified cuboidal cells (Fig. 2B-D). There was a certain amount of hyaline or mucinous material within the tubules and acini or around them. Tubules and acini were highlighted by periodic acid-Schiff stain (Fig. 2E). Immunohistochemistry for cytokeratin (CK)20, carcinoembryonic antigen, S100, thyroid transcription factor 1 and CDX-2 was negative, and thus, neuroendocrine and visceral metastatic adenocarcinoma was excluded. The tumor cells tested positive for CK7, CAM5.2, epithelial membrane antigen and CD117, and the tumor cells in the medial margin of the glandular cavity were positive for P63 (Fig. 3).

A detailed evaluation of the patient was performed. Hematological and biochemical parameters were within normal limits. Cranial MRI revealed multiple mass lesions in the right pharynx, inferior temporal fossa, middle cranial fossa and right frontotemporal subcutaneous, which was hyper-intense on T1-weighted or T2-weighted MRI. The lesion was again enhanced heterogeneously on contrast and the largest mass measured $4.0 \times 3.0 \times 3.5 \mathrm{~cm}$. In the right mastoid process, an enhanced $\mathrm{T} 2$ signal indicated the presence of right mastoid process inflammation (Fig. 4A-D). Chest CT (plain and contrast) revealed multiple metastases in both sides of the lungs (Fig. 4E) and it was hyper-densely situated in the mediastinum, suggesting calcification. Ultrasonography of the abdomen indicated no obvious abnormalities. After receiving radiotherapy one time at a local hospital, the patient exhibited no significant clinical improvement and refused further treatment. At a follow-up time of $\sim 2$ years, the patient appeared in good spirits, but refused further medical examination and treatment, and had become more frail. The patient was then lost to follow-up.

\section{Discussion}

ACC is a rare, slow-growing form of high-grade adenocarcinoma that begins in glandular tissues. ACC occurs primarily in the head and neck, most commonly in the salivary glands, and accounts for $1-2 \%$ of all cases of head and neck cancer, $\sim 10 \%$ of all salivary neoplasms and $22 \%$ of all salivary gland malignancies (3-5). ACC may also occur in other locations in the body, including the tracheobronchial tree, breast, foot and cervix (6-10). ACC of the external ear is rare, and to the best of our knowledge, only a few cases have been reported in the literature in the English language. The incidence rate ranges from 5 to $10 \%$ in the external auditory canal $(11,12)$. To date, the incidence rate of ACC of the external ear has not been reported in China. According to previous studies, ACC of the external auditory canal was most commonly diagnosed in the fifth decade of life and was more common in females $(11,13)$.

The clinical symptoms of ACC depend on the location of the tumor. As the tumor growth of ACC is frequently slow, numerous patients may live asymptomatically for a long time, even with metastatic lesions. ACC is rarely associated with regional lymph node involvement and there is only a $13 \%$ rate of nodal metastasis in patients with $\mathrm{ACC}$ of the external ear (13), which is similar to the 5-10\% rate reported for ACC in

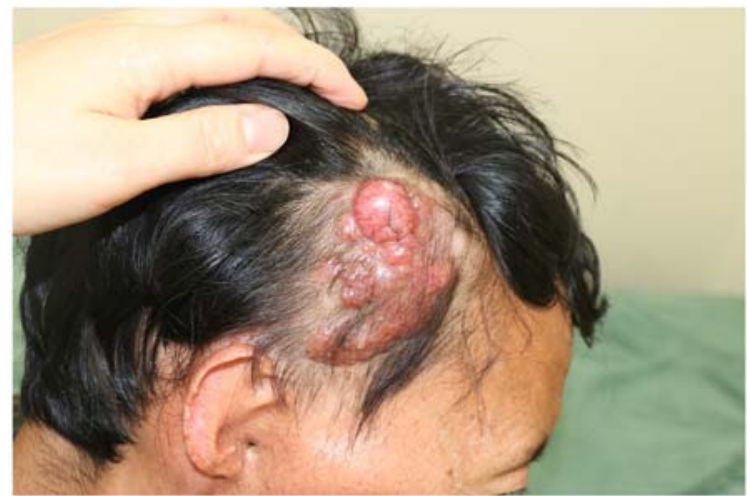

Figure 1. Image of the lesion when the patient visited The First Affiliated Hospital of Army Medical University in May 2016. On the right temple, a proliferative growth with an uneven surface and irregular margins with obviously dilated capillaries were present.

other head and neck subsites (14). According to the literature, delayed hematogenous metastases occur in $25-50 \%$ of patients with ACC and the most common locations of metastases are the lungs and bones (15). Distant metastasis frequently occurs without local recurrence. The patient described in the present case report was a 46 -year-old male. While skull base infiltration and lung metastases have been previously observed, no nodal metastasis was detected in this patient. This may be explained by the fact that ACC tends to spread along nerves, known as a perineural invasion, or through the bloodstream (15). In clinical practice, the majority of ACCs in the skin are therefore primary neoplasms. Cutaneous ACC may also represent a direct extension from an underlying salivary gland primary neoplasm. Metastasis from a more distant site has rarely been observed (10). The lesions of the patient in the present case report appeared on the right external ear at first, and subsequently, skull base and lung metastases were detected. As a consequence, the ACC on the right external ear may arise from the underlying ceruminous gland or adjacent salivary gland. ACC is known for having long periods of no growth, or indolence, followed by growth spurts. However, ACC may present as aggressive forms in certain patients, making the course of the ACC unpredictable (10). The long period of the cancer evolving was reflected in the patient of the present case report.

A biopsy is essential for the diagnosis of ACC. It has a characteristic cribiform or sieve-like pattern, in which bundles of epithelial cells surround ducts or glandular structures $(16,17)$. In cutaneous ACC, the tumor tissue is rarely attached to the epidermis or hair follicles. Mitotic activity is rare and peripheral palisading is uncommon (17). Immunohistochemistry is useful for the diagnosis of ACC $(16,18)$. ACC is characterized by slow progression but has a high propensity to invade and metastasize, particularly via perineural invasion, which occurs in $\leq 70 \%$ of ACC cases (19). ACC involving the skull base (orbital contents/bone, sinonasal cavities, nasopharynx, cavernous sinus, clivus, pterygopalatine fossa and infratemporal fossa) is usually identified via novel imaging techniques (20). Clinically, imaging techniques, primarily MRI or CT scan, are useful in order to determine the size and location of the tumor $(9,21)$, and a positron emission tomography scan may also be used to detect metastatic lesions early (22). 
A

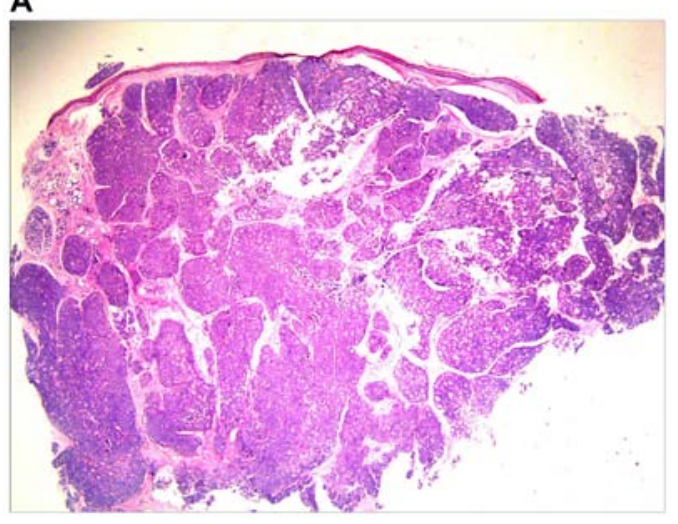

B
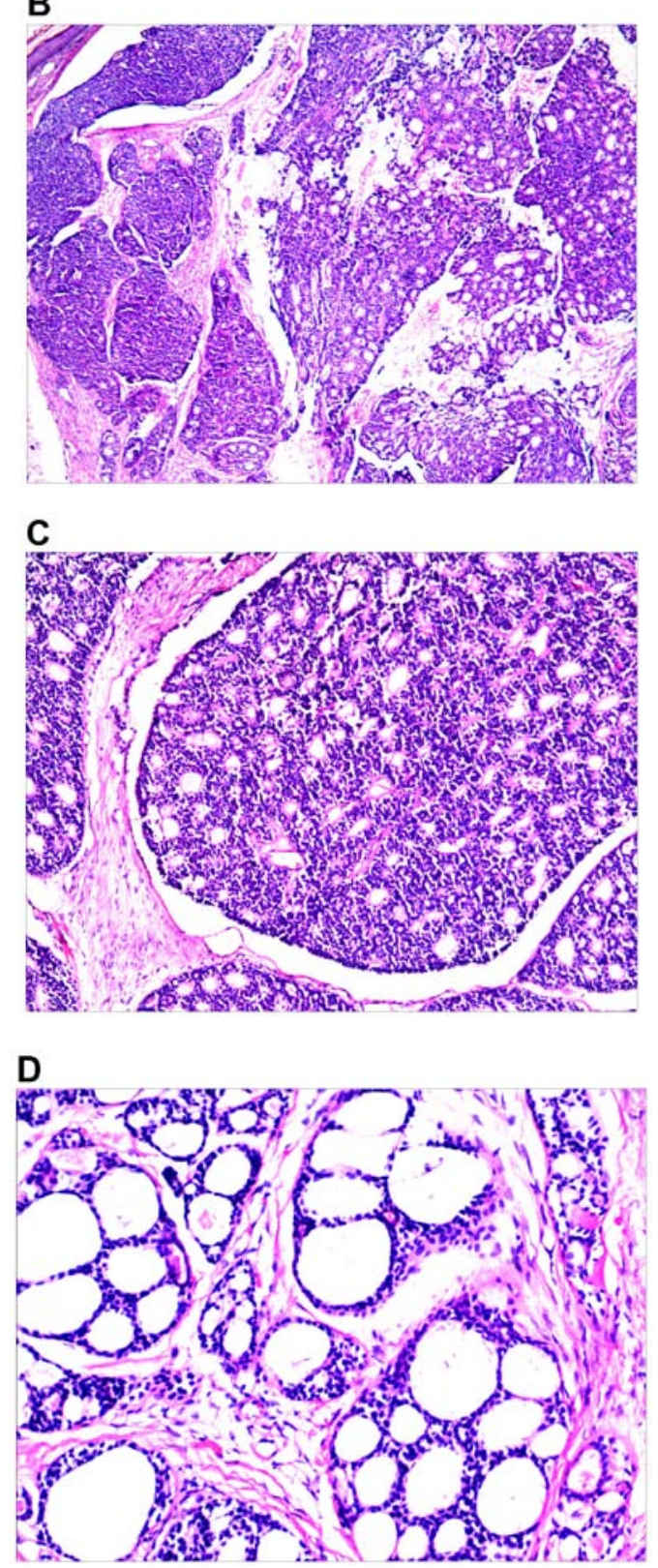

Figure 2. Histological images of the tumor. (A) Histopathology revealed a cellular tumor with sharp circumscription, and ductal differentiation and focal myxoid stroma at low power (H\&E; magnification, x12.5). (B-D) The tumor cells exhibited a tubular and characteristic cribriform growth pattern, with hyaline material within and around the tumor cells. The tumor cells appeared uniform with vesicular nuclei and prominent nucleoli. Clear cell forms and cystic spaces are evident (H\&E; magnification, $\mathrm{x} 25$ in B, $\mathrm{x} 100$ in $\mathrm{C}$, and $\mathrm{x} 200$ in D).

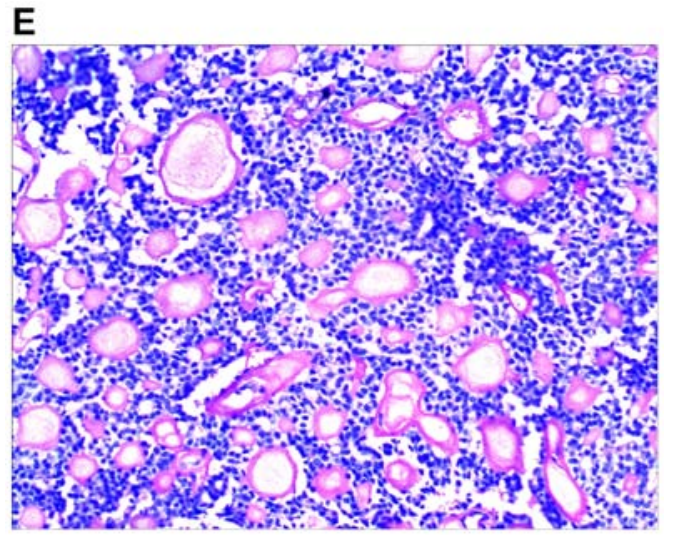

Figure 2. Continued. Histological images of the tumor. (E) The cystic spaces are highlighted by the periodic acid-Schiff stain (magnification, $\mathrm{x} 200$ ).

The primary treatment modalities are surgery and radiation therapy. Surgical removal of the tumor is the best treatment for ACC as long as it is performed safely and is likely to have a good outcome. Radiation therapy, as an adjuvant therapy following surgery, is particularly effective for ACC by eliminating microscopic cancer cells. Certain studies have indicated that surgery alone was associated with improved overall survival compared with a combination of surgery and radiotherapy, while radiotherapy alone was associated with comparatively worse overall survival (13). It is uncertain whether chemotherapy alters the natural course of metastatic ACC at any site $(23,24)$. With further study on the pathogenesis of ACC, more and more gene mutations have been detected, including epidermal growth factor receptor (EGFR), ALK receptor tyrosine kinase (25) and notch receptor 1 (NOTCH1) (26). At present, targeted therapies are being developed that may be useful in the treatment of ACC, including ALK-targeted gold nanoparticles and EGFR-tyrosine kinase inhibitor gefitinib (6,7). Previous studies have demonstrated that lung ACC is associated with EGFR mutation and that it exhibited a good response to gefitinib (6,7). Distant metastasis is a poor prognostic factor, but nodal metastasis had no impact on overall survival. Local recurrence is another common poor prognostic factor. Local recurrences are frequent and may present as early as 2 years after initial treatment, or after a number of years from the initial treatment (5). In addition, a solid pattern, higher age, black ethnicity and male sex are also poor prognostic factors (11). The survival rate for people with ACC at 5 years was reported to be $80.4-89.1 \%(20,27)$. Local recurrence and distant metastasis result in poor long-term survival, with $80-90 \%$ of patients succumbing to the disease within 10-15 years (28). According to the literature, skull base metastasis is a predictor of lower overall survival and higher rate of local recurrence (25). Further molecular-level investigations are required in order to improve the current understanding of the unique recurrence and metastasis in ACC. Recently, B-cell-specific moloney murine leukemia virus insertion region (Bmi)-1 mRNA levels were revealed to be markedly elevated in ACC tissues compared with those in the adjacent non-cancerous salivary gland tissues. Further study suggested that Bmi-1 may have a crucial role in ACC progression through interaction with the epithelial-to-mesenchymal transition-associated markers, and may predict poor survival (29). 

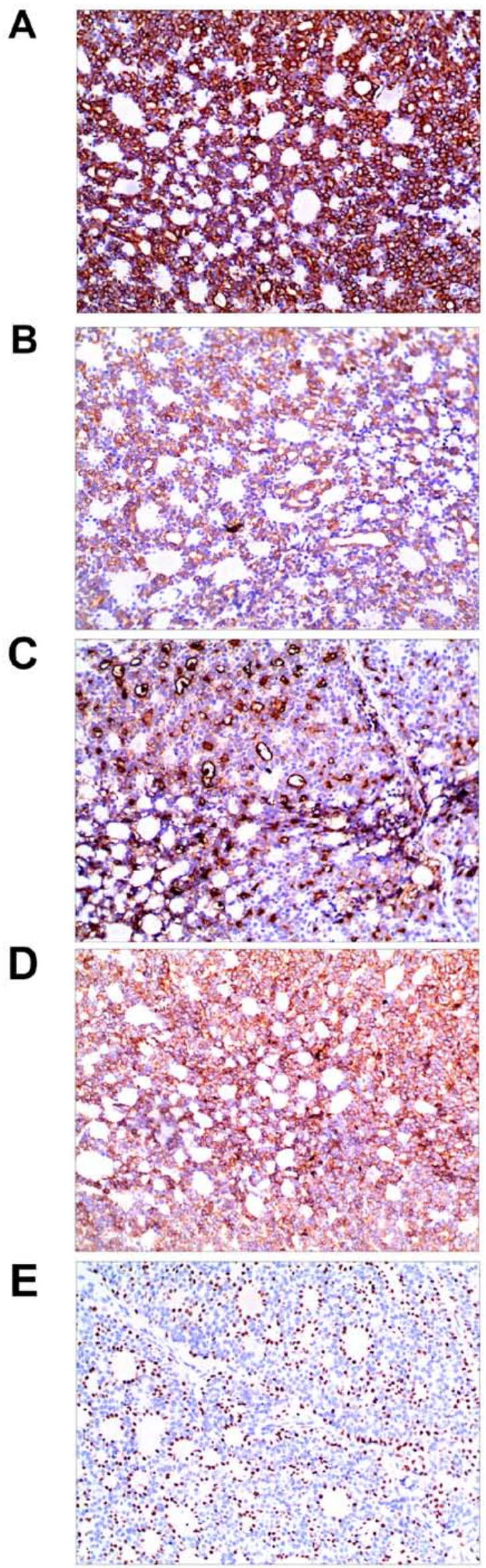

Figure 3. Immunohistochemical staining for (A) cytokeratin 7, (B) CAM5.2, (C) epithelial membrane antigen, (D) CD117 were positive in the tumor cells, and (E)the tumor cells in the medial margin of the glandular cavity were positive for P63, suggesting myoepithelial cells (SP, magnification x200).
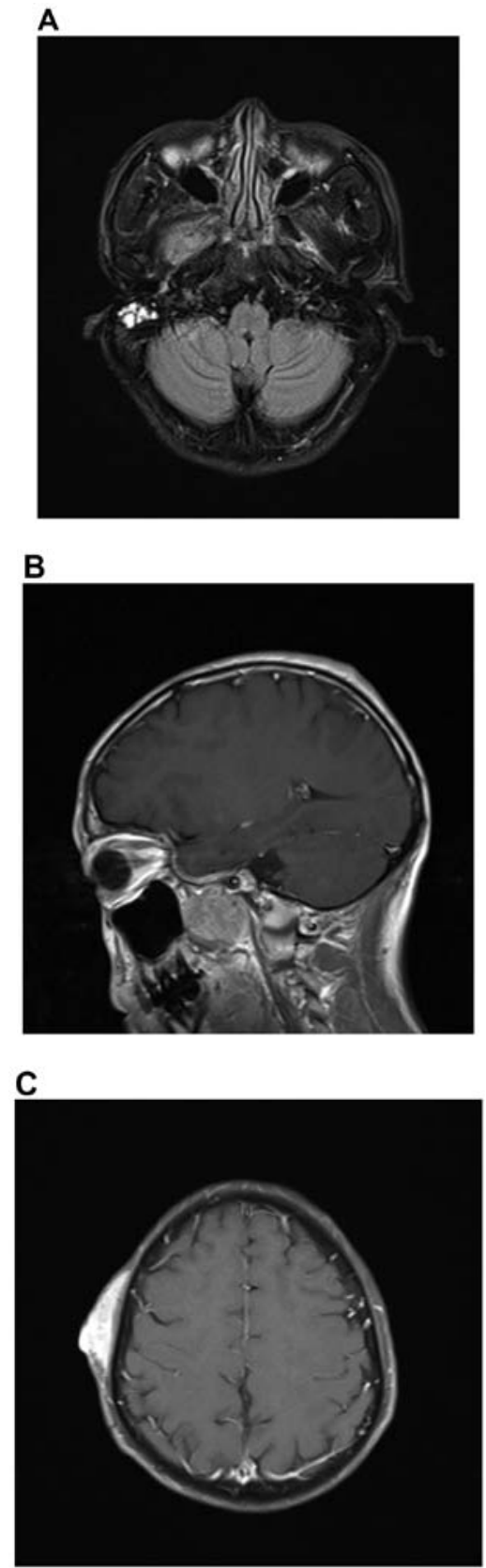

D

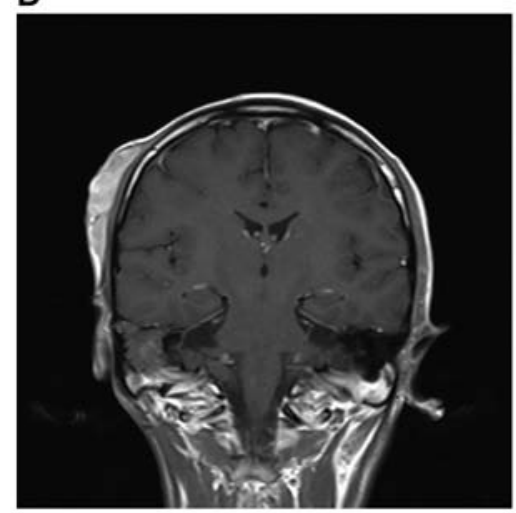

Figure 4. MRI demonstrates superior contrast resolution of multiple mass lesions in the right pharynx, inferior temporal fossa, middle cranial fossa and right frontotemporal region. (A and B) T2WI. (A) Coronal scan and (B) sagittal scan. In the right mastoid process, an enhanced T2 signal suggested inflammation of the right mastoid process. (C and D) On T1WI, the right side of the temporal region featured an enhanced $\mathrm{T} 1$ signal suggesting the presence of a subcutaneous neoplasm. (C) Coronal scan and (D) sagittal scan. 


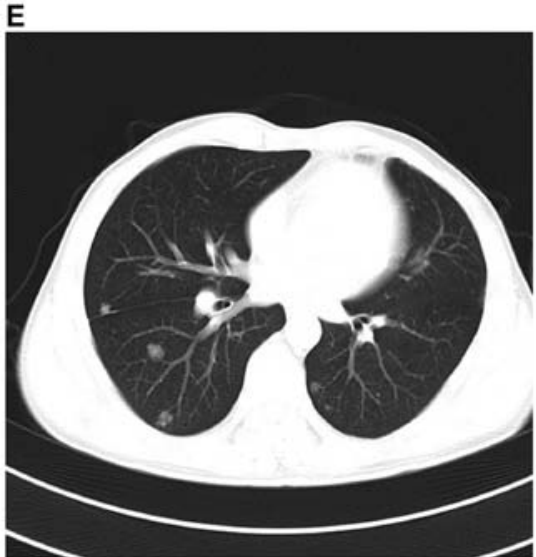

Figure 4. Continued. MRI demonstrates superior contrast resolution of multiple mass lesions in the right pharynx, inferior temporal fossa, middle cranial fossa and right frontotemporal region. (E) Chest enhanced CT scan indicated multiple metastases in the bilateral lungs. T1WI, T1-weighted imaging.

In conclusion, $\mathrm{ACC}$ is an uncommon disease that primarily arises from a salivary gland low-grade malignancy that presents with unpredictable growth and poorly understood prognostic factors. It is imperative for patients to receive an early diagnosis and surgery to remove the tumor in a timely manner, which may markedly improve the survival of these patients.

\section{Acknowledgements}

Not applicable.

\section{Funding}

The present study was partly supported by the education training research project of the First Affiliated Hospital of Army Medical University (grant no. SWH2015JY07y to ZZ).

\section{Availability of data and materials}

The datasets used and/or analyzed during the current study are available from the corresponding author on reasonable request.

\section{Authors' contributions}

$\mathrm{ZZ}$ was responsible for the conception, design, content and writing of the manuscript. JH was responsible for acquisition, analysis and interpretation of the images. YY made substantial contributions to conception,design, drafting and revising the manuscript. XY made substantial contributions to acquisition, analysis and interpretation of data and revising the manuscript critically for important intellectual content. ZS made substantial contributions to conception and design, revising and proofreading the manuscript and gave final approval of the version to be published. All authors have read and approved the final manuscript.

\section{Ethics approval and consent to participate}

Ethics approval for the present study was obtained from the Ethics Committee of the Southwest Hospital of Chongqing
(The First Affiliated Hospital of Army Medical University; Chongqing, China). Informed consent was obtained from the patient.

\section{Patient consent for publication}

The patient provided written informed consent for publication of the present case report.

\section{Competing interests}

The authors declare that they have no competing interests.

\section{References}

1. Ko JJ, Siever JE, Hao D, Simpson R and Lau HY: Adenoid cystic carcinoma of head and neck: Clinical predictors of outcome from a Canadian centre. Curr Oncol 23: 26-33, 2016.

2. Ellington CL, Goodman M, Kono SA, Grist W, Wadsworth T, Chen AY, Owonikoko T, Ramalingam S, Shin DM, Khuri FR, et al: Adenoid cystic carcinoma of the head and neck: Incidence and survival trends based on 1973-2007 surveillance, epidemiology, and end results data. Cancer 118: 4444-4451, 2012.

3. Brookstone MS, Huvos AG and Spiro RH: Central adenoid cystic carcinoma of the mandible. J Oral Maxillofac Surg 48: 1329-1333, 1990

4. Zhang CY, Xia RH, Han J, Wang BS, Tian WD, Zhong LP, Tian Z, Wang LZ, Hu YH and Li J: Adenoid cystic carcinoma of the head and neck: Clinicopathologic analysis of 218 cases in a Chinese population. Oral Surg Oral Med Oral Pathol Oral Radiol 115: 368-375, 2013.

5. Bradley PJ: Adenoid cystic carcinoma of the head and neck: A review. Curr Opin Otolaryngol Head Neck Surg 12: 127-132, 2004

6. Fujita M, Matsumoto T, Hirano R, Uchino J, Hirota T, Yamaguchi E, Kubo A, Yokoi T, Nabeshima K and Watanabe K: Adenoid cystic carcinoma of the lung with an EGFR mutation. Intern Med 55: 1621-1624, 2016.

7. Huo Z, Wu H, Li S and Liang Z: Molecular genetic studies on EGFR, KRAS, BRAF, ALK, PIK3CA, PDGFRA, and DDR2 in primary pulmonary adenoid cystic carcinoma. Diagn Pathol 10: $161,2015$.

8. Agunbiade MM,Lalehparvar S and Khaladj M: Cutaneous adenoid cystic carcinoma with perineural invasion diagnosed in the foot: A case report. J Am Podiatr Med Assoc 107: 457-460, 2017.

9. Treitl D, Radkani P, Rizer M, EI Hussein S, Paramo JC and Mesko TW: Adenoid cystic carcinoma of the breast, 20 years of experience in a single center with review of literature. Breast Cancer 25: 28-33, 2018.

10. Rais M, Kharmoum J, Ech-Charif S and EI Khannoussi B: Adenoid cystic carcinoma of the uterine cervix: A report of 2 cases. Case Rep Pathol 2017: 8401741, 2017.

11. Dong F, Gidley PW, Ho T, Luna MA, Gingsberg LE and Sturgis FM: Adenoid cystic carcinoma of external auditory canal. Laryngoscope 118: 1591-1596, 2008.

12. Ouaz K, Robier A, Lescanne E, Bobillier C, Morinière S and Bakhos D: Cancer of the external auditory canal. Eur Ann Otorhinolaryngol Head Neck Dis 130: 175-182, 2013.

13. Green RW and Megwalu UC: Adenoid cystic carcinoma of the external ear: A population based study. Am J Otolaryngol 37: 346-350, 2016

14. Min R, Siyi L, Wenjun Y, Ow A, Lizheng W, Minjun D and Chenping Z: Salivary gland adenoid cystic carcinoma with cervical lymph node metastasis: A preliminary study of 62 cases. Int J Oral Maxillofac Surg 41: 952-927, 2012.

15. Ferlito A, Shaha AR, Silver CE, Rinaldo A and Mondin V: Incidence and sites of distant metastases from head and neck cancer. ORL J Otorhinolaryngol Relat Spec 63: 202-207, 2001.

16. Arps DP, Chan MP, Patel RM and Andea AA: Primary cutaneous cribriform carcinoma: Report of six cases with clinicopathologic data and immunohistochemical profile. J Cutan Pathol 42: 379-387, 2015.

17. Ramakrishnan R, Chaudhry IH, Ramdial P, Lazar AJ, McMenamin ME, Kazakov D, Brenn T and Calonje E: Primary cutaneous adenoid cystic carcinoma: A clinicopathologic and immunohistochemical study of 27 cases. Am J Surg Pathol 37: 1603-1611, 2013 
18. van Weert S, Bloemena E, van der Waal I, de Bree R, Rietveld DH Kuik JD and Leemans CR: Adenoid cystic carcinoma of the head and neck: A single-center analysis of 105 consecutive cases over a 30-year period. Oral Oncol 49: 824-829, 2013.

19. Alkan BI,Bozdogan O,KaradenizMandBozdoğan N: Twodifferent cell populations is an important clue for diagnosis of primary cutaneous adenoid cystic carcinoma: Immunohistochemical study. Case Rep Pathol 2017: 7949361, 2017.

20. Jang S, Patel PN, Kimple RJ and McCulloch TM: Clinical outcomes and prognostic factors of adenoid cystic carcinoma of the head and neck. Anticancer Res 37: 3045-3052, 2017.

21. Kumar PP, Patil AA, Ogren FP, Johansson SL and Reeves MA: Intracranial skip metastasis from parotid and facial skin tumors: Mechanism, diagnosis, and treatment. J Natl Med Assoc 85: 369-374, 1993.

22. Girelli L, Locati L, Galeone C, Scanagatta P, Duranti L, Licitra L and Pastorino U: Lung metastasectomy in adenoid cystic cancer: Is it worth it? Oral Oncol 65: 114-118, 2017.

23. Honings J, van Dijck JA, Verhagen AF, van der Heijden HF and Marres HA: Incidence and treatment of tracheal cancer: A nationwide study in the Netherlands. Ann Surg Oncol 14: 968-969, 2007.

24. Laurie SA, Ho AL, Fury MG, Sherman E and Pfister DG: Systemic therapy in the management of metastatic or locally recurrent adenoid cystic carcinoma of the salivary glands: A systematic review. Lancet Oncol 12: 815-824, 2011.

25. Hazkani I, Motiei M, Betzer O, Sadan T, Bragilovski D, Lubimov L, Mizrachi A, Hadar T, Levi M, Ben-Aharon I, et al: Can molecular profiling enhance radiotherapy? Impact of personalized targeted gold nanoparticles on radiosensitivity and imaging of adenoid cystic carcinoma. Theranostics 7: 3962-3971, 2017.
26. Ferrarotto R, Mitani Y, Diao L, Guijarro I, Wang J, Zweidler-McKay P, Bell D, William WN Jr, Glisson BS, Wick MJ, et al: Activating NOTCH1 mutations define a distinct subgroup of patients with adenoid cystic carcinoma who have poor prognosis, propensity to bone and liver metastasis, and potential responsiveness to Notch1 inhibitors. J Clin Oncol 35: 352-360, 2017.

27. Cao C, Ge M, Chen X, Xu J and Chen C: Clinical outcomes and prognostic factors of salivary gland adenoid cystic carcinomas: A case control study. Oral Surg Oral Med Oral Pathol Oral Radiol 123: 531-535, 2017.

28. Thompson L: World health organization classification of tumours: Pathology and genetics of head and neck tumours. Ear Nose Throat J 85: 74, 2006.

29. Yi C, Li BB and Zhou CX: Bmi-1 expression predicts prognosis in salivary adenoid cystic carcinoma and correlates with epithelial-mesenchymal transition-related factors. Ann Diagn Pathol 22: 38-44, 2016.

This work is licensed under a Creative Commons Attribution-NonCommercial-NoDerivatives 4.0 International (CC BY-NC-ND 4.0) License. 